\title{
Численное пленочное моделирование электромагнитного поля на Воронежском кристаллическом массиве и сопредельных структурах
}

\author{
(C2021 В. Н. Груздев ${ }^{凶}$ \\ Воронежский государственный университет, \\ Университетская пл. 1, 394018 Воронеж, Российская федерация
}

\begin{abstract}
Аннотация
Введение: Вариации естественного электромагнитного поля позволяют изучать глубинное строение Воронежского кристаллического массива и сопряженных структур. На характер наблюденного геомагнитного поля оказывают влияние хорошо проводящие породы осадочного чехла

Методика: Для учета искажений наблюдаемого магнитотеллурического поля в пределах Воронежского кристаллического массива, Рязано-Саратовского прогиба, Днепрово-Донецкой впадины региональными гальваническими и индукционными эффектами выполнено численное пленочное моделирование. Осадочный чехол данных структур аппроксимировался пленкой Прайса-Шейнмана с заданной суммарной продольной проводимостью $\mathrm{S}(\mathrm{x}, \mathrm{y})$.

Результаты и обсуждение: Под действием неоднородного проводящего осадочного чехла горизонтальные компоненты электрического поля и вертикальный компонент магнитного поля сильно искажается, а горизонтальные компоненты переменного магнитного поля значительно меньше подвержены влиянию проводящего осадочного чехла.

Заключение: Учет искажений переменного электромагнитного поля проводящим осадочным чехлом позволил оценить аномалии электропроводности, выявленные по магнитовариационным и магнитотеллурическим данным в пределах ВКМ и сопряженных структур на различных уровнях земной коры.
\end{abstract}

Ключевые слова: Воронежский кристаллический массив, численное пленочное моделирование, осадочный чехол, гальванические и индукционные искажения

Источник финансирования: грант РФФИ 19-05-00336

Для циттирования: Груздев В. Н. Численное пленочное моделирование электромагнитного поля на Воронежском кристаллическом массиве и сопредельных структурах // Вестник Воронежского государственного университета. Серия: Геология. 2021. №3. С. 79-86.

DOI: https://doi.org/10.17308/geology.2021.3/3652

\section{Введение}

Вариации естественного электромагнитного поля по $\mathrm{H}_{\mathrm{x}}, \mathrm{H}_{\mathrm{y}}, \mathrm{H}_{\mathrm{z}}, \mathrm{E}_{\mathrm{x}}, \mathrm{E}_{\mathrm{y}}$ составляющим позволяют изучать глубинное строение Воронежского кристаллического массива (ВКМ) и сопряженных структур Рязано-Саратовского прогиба (РСП), а также Днепрово-Донецкой впадины (ДДВ). Для решения данной задачи применялись методы магнитовариационного профилирования (МВП) и магнитотеллурического зондирования (МТЗ). По определению: $\mathrm{E}_{\mathrm{x}}$ компонента электрического поля и $\mathrm{H}_{\mathrm{x}}$ компонента магнитного поля представлены в направлении магнитного меридиана; Е компонента электрического

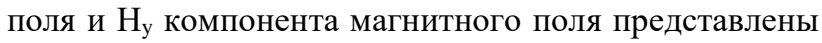
в направлении магнитной широты; $\mathrm{H}_{\mathrm{z}}$ компонента характеризует изменение магнитного поля по вертикальному направлению. Известно, что на вариации наблюдённого геомагнитного поля оказывают влияние хорошо проводящие породы осадочного чехла $[1,2,3]$.

Контент доступен под лицензией Creative Commons Attribution 4.0 License.

\footnotetext{
$\bowtie$ Груздев Владислав Николаевич, e-mail: grumerr@rambler.ru
} 
Для определения характера искажений вариаций электромагнитного поля горизонтальными и вертикальными неоднородностями электрического сопротивления осадочного чехла ВКМ и прилегающих структур применялось численного пленочного моделирования $[4,5]$.

\section{Методика пленочного моделирования}

Для учета искажений магнитотеллурического поля в пределах ВКМ, РСП, ДДВ региональными гальваническими и индукционными эффектами численное пленочное моделирование осуществлялась на территории размером 2200 км на 1100 км, включающей также структуры Предкавказа, Украинский щит, Белорусский массив, Крестцово-Волдайский прогиб, Токмовский свод, Прикаспийскую впадину, Московскую синеклизу (рис. 1) [6, 7]. Осадочный чехол указанных структур аппроксимировался пленкой Прайса Шейнмана с суммарной продольной проводимостью $\mathrm{S}(\mathrm{x}, \mathrm{y})$, меняющейся только в горизонтальных направлениях. Предполагалось, что осадочный чехол подстилается идеальным изолятором, поэтому перетеканием электрических токов из осадочного чехла в проводящий кристаллический фундамент пренебрегалось, что естественно внесло некоторые погрешности в результаты моделирования [6,7].

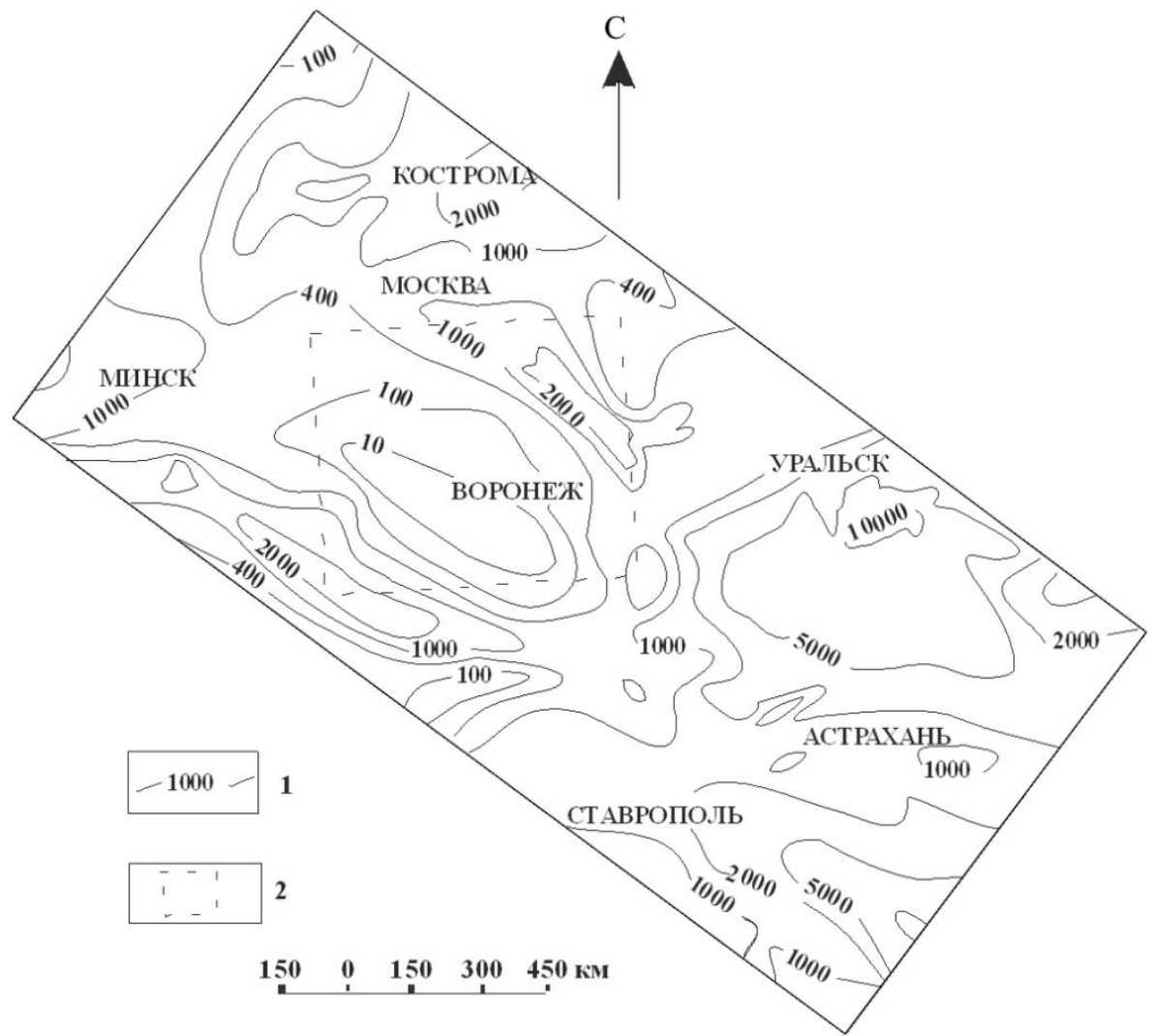

Рис. 1. Схема суммарной продольной проводимости осадочного чехла (S) масштаба 1:5000000 по данным публикаций [6, 7]. Условные обозначения: 1 - изолинии $\mathrm{S}$ в См; 2 - участок, по которому выполнен анализ результатов численного пленочного моделирования.

[Fig. 1. Diagram of the total longitudinal conductivity of the sedimentary cover (S) on a scale of 1:5,000,000 according to publications [6, 7]. Legend: (1) - isolines S in cm; (2) - a section along which the results of numerical film modelling were analysed. Translation symbols from the тар: Минск-Minsk, Москва-Моscow, Кострома-Kоstroта, Воронеж-Voronezh, Уральск-Uralsk, Acmpaxaнь-Astrakhan, Ставрополь - Stavropol, км-km.]

Для проведении численного пленочного моделирования для большинства структур значения $\mathrm{S}(\mathrm{x}, \mathrm{y})$ получены с карты масштаба 1:5000000 для Восточно-Европейской платформы. Непосредственно для структур ВКМ, РСП, северо-восточного борта ДДВ использовались более детальные данные о распределении суммарной продольной проводимости $\mathrm{S}(\mathrm{x}, \mathrm{y})$ с карты масштаба 1:1000000. Вся рассматриваемая территория разбивалась на ячейки размеров 35 км х 35 км, в пределах которых усреднялись значения $\mathrm{S}(\mathrm{x}, \mathrm{y})$ (рис.1) [6, 7, 8, 9 ]. За пределами территории моделирования нормальный фон $\mathrm{S}(\mathrm{x}, \mathrm{y})$ принят равным 400 См, что является его реальным значением вблизи структуры ВКМ, завышенным в 4 раза на северо-западной и западной границах рассматриваемой территории и заниженным в 10-20 раз на юговосточной границе. Несоответствие реальных и принятых для моделирования значений фона $\mathrm{S}(\mathrm{x}, \mathrm{y})$ на больших удалениях от ВКМ практически слабо сказывается на характере моделируемого магнитотеллурического поля в пределах ВКМ, РСП и ДДВ. 
Численное пленочное моделирование выполнено для периодов Т =900, 1800, 5400, 10800, 43200 с при X - и Y - поляризациях первичного электрического поля. $\mathrm{X}$ - поляризация соответствует направлению в крест преобладающего простирания изолиний $\mathrm{S}$ на территории ВКМ, РСП и ДДВ, а Y - поляризация соответствует региональному простиранию изолиний $\mathrm{S}$ в пределах тех же структур. В процедуре моделирования рассчитывались нормированные относительно базы амплитуды и фазы $\mathrm{E}_{\mathrm{x}}$, и $\mathrm{E}_{\mathrm{y}}$ компонент электрического, a также $\mathrm{H}_{\mathrm{x}}, \mathrm{H}_{\mathrm{y}}, \mathrm{H}_{\mathrm{z}}$ компонент магнитного полей, используемые при экспериментальных наблюдениях. На основании этих данных вычислялись зависимости кажущегося сопротивления $\rho_{к}$ от частоты.

\section{Результаты}

Под действием неоднородного проводящего осадочного чехла по данным численного пленочного моделирования электрическое поле искажается. Значения нормированного модуля горизонтальных $\mathrm{E}^{\mathrm{x}}{ }_{\mathrm{x}}$ и $\mathrm{E}_{\mathrm{y}}^{\mathrm{y}}$ компонент (где верхний индекс х указывает северовосточное направление поляризации первичного электрического поля, а индекс у на северо-западное направление поляризации первичного поля) на своде ВКМ меняются от 1.5 до 2.0, а на северо-западном и юго-восточном его склонах достигают величины 3.0. В наиболее погруженных частях РСП и ДДВ отмечается уменьшение значений нормированных модулей $\mathrm{E}_{\mathrm{x}}^{\mathrm{x}}$ и

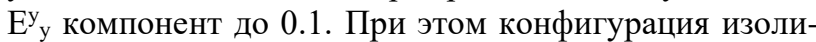
ний модулей $\mathrm{E}_{\mathrm{x}}^{\mathrm{x}}$ и $\mathrm{E}_{\mathrm{y}}^{\mathrm{y}}$ компонент совпадает с конфигурацией изолиний суммарной продольной проводимости $\mathrm{S}(\mathrm{x}, \mathrm{y})$, особенно на склонах ВКМ (рис. 2). Фаза $\mathrm{E}_{\mathrm{x}}^{\mathrm{x}}$ компоненты меняется от $165^{\circ}$ (в пределах ВКМ) до $172^{0}$ (на территории РСП и ДДВ), а Е у компонента - от $348^{0}$ (для территории ВКМ) до $360^{\circ}$ (в области РСП и ДДВ) $[10,11,12]$.

Нормированный модуль горизонтальных электрических $\mathrm{E}_{\mathrm{x}}^{\mathrm{y}}$ и $\mathrm{E}_{\mathrm{y}}^{\mathrm{x}}$ компонент для указанных поляризаций первичного электрического поля изменяется в пределах 0.4 - 1.0 на северо-западном и юго-восточном склонах ВКМ, а форма изолиний приобретает изометрический вид. В сводовой части ВКМ и в пределах РСП и ДДВ значения модулей $\mathrm{E}_{\mathrm{x}}^{\mathrm{y}}$ и $\mathrm{E}_{\mathrm{y}}^{\mathrm{x}}$ не превышает величины 0.1. На своде ВКМ отмечаются большие градиенты изменения фаз $\mathrm{E}_{\mathrm{x}}^{\mathrm{y}}$ и $\mathrm{E}_{\mathrm{y}}^{\mathrm{x}}$ компонент от $0^{0}$ до $360^{\circ}$. Пространственные фазовые различия в горизонтальных компонентах электрического поля сглаживаются с ростом периода от 900 с до 43200 с, а характер распределения модулей этих компонент не изменяется (рис. 2) $[10,11,12]$.

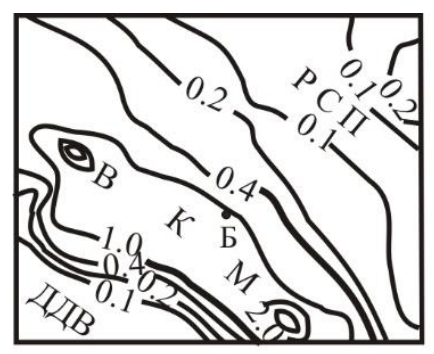

a

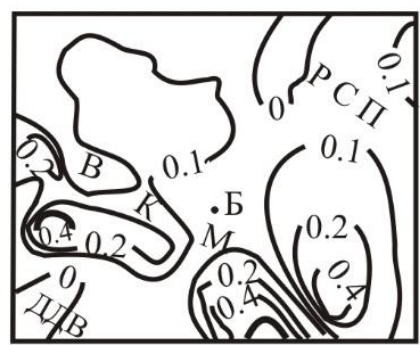

c

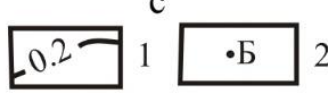

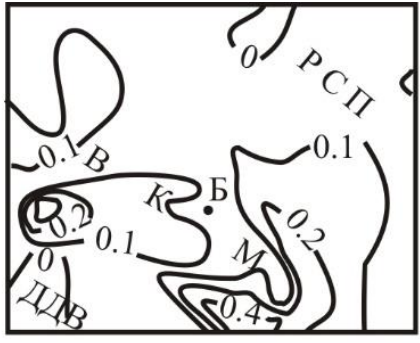

b

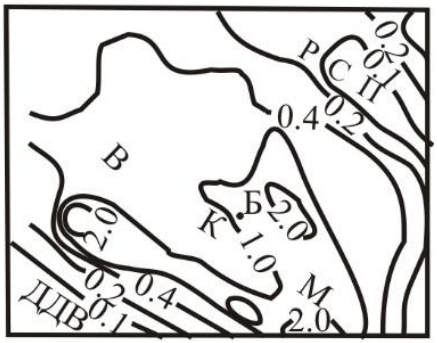

d

$100 \quad 0 \quad 100 \quad 200 \quad 300 \quad 400$ км
Рис. 2. Распределение электрического поля на территории ВКМ и сопредельных структур по данным численного пленочного моделирования $(\mathrm{T}=1800 \mathrm{c})$ : a) $\left.\left|\mathrm{E}_{\mathrm{x}} \mathrm{x}\right|, \mathrm{b}\right)\left|\mathrm{E}_{\mathrm{y}}^{\mathrm{x}}\right|$ при поляризации первичного электрического поля в северовосточном направлении (ось х); b) $\left|\mathrm{E}_{\mathrm{x}} \mathrm{y}\right|$, d) $\left|\mathrm{E}_{\mathrm{y}} \mathrm{y}\right|$ при поляризации первичного электрического поля в северо-западном направлении (ось у). Условные обозначения: 1 - изолинии модуля горизонтальных компонент электрического поля в нормированных единицах; 2 - базисный пункт наблюдения вариаций геомагнитного поля.

[Fig. 2. Distribution of the electric field on the territory of the VCM and adjacent structures according to the data of numerical film modelling ( $\mathrm{T}$ = $1800 \mathrm{~s})$ : a) $\left|\mathrm{E}_{\mathrm{x}} \mathrm{x}\right|$, b) $\left|\mathrm{E}_{\mathrm{y}} \mathrm{x}\right|$ when the primary electric field is polarised in the north-east direction (x-axis); c) $\left|E_{x} y\right|$, d) $\left|E_{y} y\right|$ when the primary electric field is polarised in the north-west direction (y-axis). Legend: (1) - isolines of the modulus of the horizontal components of the electric field in normalised units; (2) - the basic point of observation of variations in the geomagnetic field. Translation symbols from the map: $B K M-V C M$, ДДВ - DDR, РCП-RST, км-km.]
Горизонтальные составляющие переменного магнитного поля $\left(\mathrm{H}_{\mathrm{y}}, \mathrm{H}_{\mathrm{x}}\right)$ для рассматриваемых периодов значительно меньше подвержены влиянию проводящего осадочного чехла. Величина нормированного модуля $\mathrm{H}_{\mathrm{x}}^{\mathrm{y}}$ - компонента в пределах РСП и ДДВ равна $0.25-0.30$ для $\mathrm{T}=900$ с и 0.15 для $\mathrm{T}=1800 \mathrm{c}$, тогда как величина нормированных модулей $\mathrm{H}_{\mathrm{y}}^{\mathrm{y}}-, \mathrm{H}_{\mathrm{y}}^{\mathrm{x}}-, \mathrm{H}_{\mathrm{x}}^{\mathrm{x}}$ компонент не превышают 0.20 для $\mathrm{T}=900$ с и 0.10 для $\mathrm{T}=1800$ с (рис. 3) [10, 11].

В пределах РСП и ДДВ изменения суммарной проводимости осадочного чехла практически не сказываются на модулях горизонтальных компонент магнитного поля на периодах 5400 с и 43200 с.

На периоде $900 \mathrm{c}$ фазы $\mathrm{H}_{\mathrm{x}}^{\mathrm{x}}$ и $\mathrm{H}_{\mathrm{y}}^{\mathrm{y}}$ компонент имеют довольно сложный характер распределения (от $0^{0}$ до $\left.360^{\circ}\right)$, тогда как фаза $\mathrm{H}_{\mathrm{x}}^{\mathrm{y}}$ компонента изменяется от $230^{\circ}$ (на территории ВКМ) до $217^{0}$ (в области РСП и ДДВ), а фаза $\mathrm{H}_{\text {у }}^{\mathrm{x}}$ компонента - от $228^{0}$ (на территории ВКМ) до $220^{\circ}$ (в области РСП и ДДВ). С ростом периода в пределах структур ВКМ, РСП, ДДВ фазовые различия у горизонтальных компонент переменного магнитного поля сглаживаются $[10,11,12]$. 


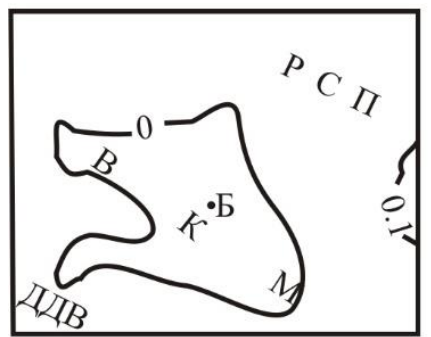

a

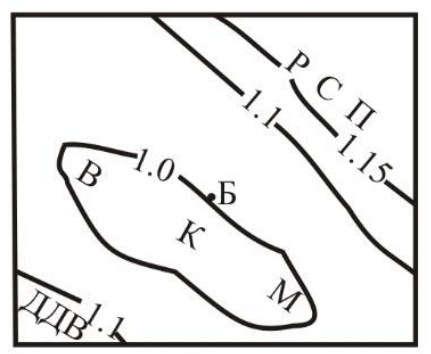

C
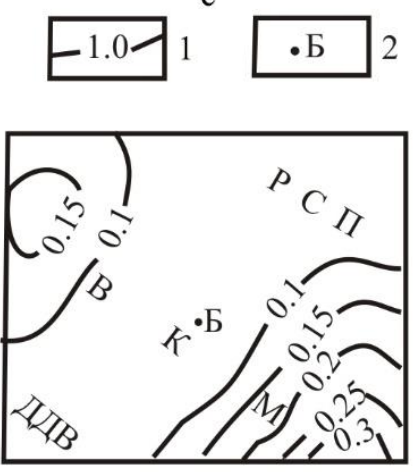

a

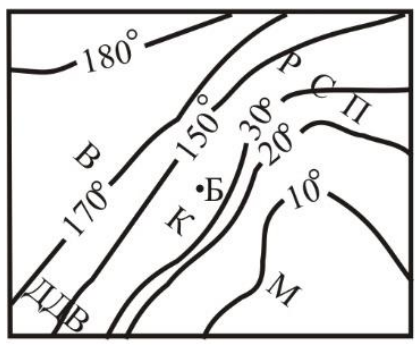

c

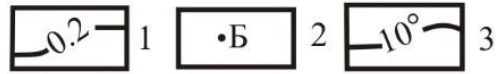

$100 \quad 0 \quad 100 \quad 200 \quad 300 \quad 400$ км

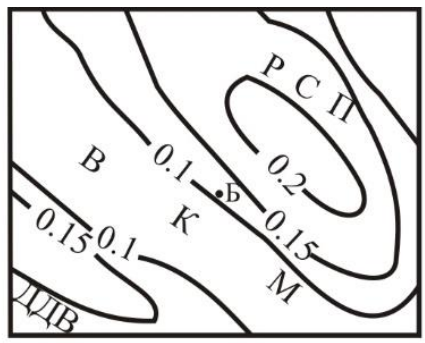

b

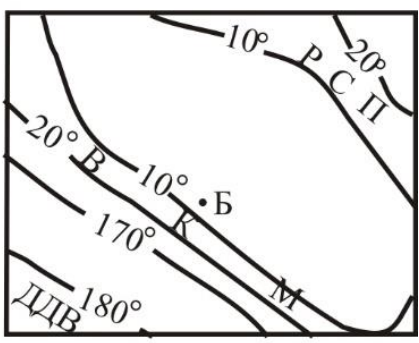

d
Рис. 3. Распределение горизонтальных составляющих магнитного поля на территории ВКМ и сопредельных структур по данным численного пленочного моделирования ( $\mathrm{T}=1800$ c): a) $\left|\mathrm{H}_{\mathrm{x}}{ }^{\mathrm{x}}\right|$, b) $\left|\mathrm{H}_{\mathrm{y}}{ }^{\mathrm{x}}\right|$ при поляризации первичного электрического поля в северо-восточном направлении (ось $\mathrm{x}$ ); c) $\left.\left|\mathrm{H}_{\mathrm{x}} \mathrm{y}\right|, \quad \mathrm{d}\right) \quad\left|\mathrm{H}_{\mathrm{y}} \mathrm{y}\right|$ при поляризации первичного электрического поля в северо-западном направлении (ось у). Условные обозначения: 1 - изолинии модуля горизонтальных компонент магнитного поля в нормированных единицах; 2 - базисный пункт наблюдения вариаций геомагнитного поля.

[Fig. 3. Distribution of the horizontal components of the magnetic field on the territory of the VCM and adjacent structures according to the data of numerical film modelling $(\mathrm{T}=1800 \mathrm{~s})$ : a) $\left|\mathrm{H}_{\mathrm{x}}{ }^{\mathrm{x}}\right|$, b) $\left|\mathrm{H}_{\mathrm{y}}{ }^{\mathrm{x}}\right|$ when the primary electric field is polarised in the north-east direction (x-axis); c) $\left|\mathrm{H}_{\mathrm{x}} \mathrm{y}\right|$, d) $\left|\mathrm{H}_{\mathrm{y}} \mathrm{y}\right|$ when the primary electric field is polarised in the north-west direction (y-axis). Legend: (1) - isolines of the modulus of the horizontal components of the magnetic field in normalised units; (2) - the basic point of observation of variations in the geomagnetic field.]

Рис. 4. Распределение вертикальной составляющей магнитного поля на территории ВКМ и сопредельных структур по данным численного пленочного моделирования ( $\mathrm{T}=1800 \mathrm{c})$ : a) $\left|\mathrm{H}_{\mathrm{z}}{ }^{\mathrm{x}}\right|$ при поляризации первичного электрического поля в северо-восточном направлении (ось х); b) $\left|\mathrm{H}_{\mathrm{z}}{ }^{\mathrm{y}}\right|$ при поляризации первичного электрического поля в северо-западном направлении (ось у); с) фаза $\mathrm{H}_{\mathrm{z}}^{\mathrm{x}}$ при поляризации первичного электрического поля в северо-восточном направлении (ось х); d) фаза $\mathrm{H}_{\mathrm{z}}^{\mathrm{y}}$ при поляризации первичного электрического поля в северо-западном направлении (ось у). Условные обозначения: 1 - изолинии модуля вертикальной составляющей магнитного поля в нормированных единицах; 2 - базисный пункт наблюдения вариаций геомагнитного поля; 3 - изолинии фазы вертикальной составляющей магнитного поля в градусах.

[Fig. 4. Distribution of the vertical component of the magnetic field on the territory of the VCM and adjacent structures according to the data of numerical film modelling ( $\mathrm{T}=1800 \mathrm{~s})$ : a) $\left|\mathrm{H}_{\mathrm{z}}{ }^{\mathrm{x}}\right|$ when the primary electric field is polarised in the north-east direction ( $\mathrm{x}$ axis); b) $\left|\mathrm{H}_{\mathrm{z}} \mathrm{y}\right|$ when the primary electric field is polarised in the north-west direction (y-axis); c) phase $\mathrm{H}_{\mathrm{z}}^{\mathrm{x}}$ when the primary electric field is polarised in the north-east direction (x-axis); d) phase $\mathrm{H}_{\mathrm{z}}{ }^{\mathrm{y}}$ when the primary electric field is polarised in the north-west direction (y-axis). Legend: $(1)$ - isolines of the modulus of the vertical component of the magnetic field in normalised units; (2) - basic point of observation of variations in the geomagnetic field; (3) - isolines of the phase of the vertical component of the magnetic field in degrees.]

Проводящий осадочный чехол оказывает сильное влияние на вертикальный компонент переменного магнитного поля $\left(\mathrm{H}_{\mathrm{z}}\right)$. Величина модуля $\mathrm{H}_{\mathrm{z}}^{\mathrm{x}}$ компонента, нормированного относительно горизонтального компонента $\left(\mathrm{H}_{\mathrm{x}}\right)$ первичного магнитного поля, на северо-западном и юго-восточном склонах ВКМ изменяется от 0.25 до $0.45(\mathrm{~T}=900 \mathrm{c})$ и от 0.20 до 0.30 $(\mathrm{T}=1800 \mathrm{c})$. В сводовой части ВКМ, в РСП и ДДВ модуль $\mathrm{H}_{\mathrm{z}}^{\mathrm{x}}$ не превышает величины 0.10. Величина модуля $\mathrm{H}_{\mathrm{z}}^{\mathrm{z}}$ компонента, нормированного относитель- но горизонтального компонента $\left(\mathrm{H}_{\mathrm{y}}\right)$, возрастает внутри впадин до $0.40(\mathrm{~T}=900 \mathrm{c})$ и до $0.20(\mathrm{~T}=1800 \mathrm{c})$ (рис. 4) [10, 11, 12].

В целом модуль $\mathrm{H}_{\mathrm{z}}$ компонента при $\mathrm{x}$ и $\mathrm{y}$ поляризациях первичного поля на периодах 5400 с и 43200 с в пределах структур ВКМ, РСП и ДДВ не превышает $10 \%$ от модуля горизонтальной составляющей. В средней части структур ВКМ, РСП и ДДВ располагается зона смены знака $\mathrm{H}^{\mathrm{x}}{ }_{\mathrm{z}}$ компонента шириной более 100 км (рис. 4а). Вдоль свода ВКМ, по 
его простиранию, отмечается зона смены знака $\mathrm{H}^{\mathrm{y}}$ компонента шириной более 40 км (рис. 4б) [10, 11].

Кроме того, был проанализирован характер поведения больших осей полярных диаграмм модулей магнитных параметров, связывающих вертикальную и горизонтальные составляющие модельного магнитного поля, что также отражает влияние изменений проводимости осадочного чехла. Малые значения этих величин для $\mathrm{T}=1800 \mathrm{c}$ в сводовой части ВКМ указывает на сильное ослабление переменного магнитного поля, создаваемого проводящими осадками, а также на суперпозицию магнитных полей от проводящих осадков РСП и ДДВ. Большие оси полярных диаграмм магнитных параметров достигают значительной величины на северо-восточном склоне ВКМ в результате влияния мощной толщи осадков РСП, на юго-западном склоне за счёт воздействия проводящих ещё более мощного комплекса осадков ДДВ. В южной и восточной частях ВКМ аналогичное влияние оказывает Прикаспийская впадина. Большие оси полярной диаграммы направлены, в основном, перпендикулярно простиранию изолиний суммарной электропроводности $\mathrm{S}$, оконтуривая сводовую часть структуры ВКМ [10, 11, 12].

В результате численного пленочного моделирования электромагнитного поля в диапазоне периодов от 900 с до 43200 с получены кривые $\rho_{\mathrm{K}}^{\text {ху м }}$ и $\rho_{\mathrm{K}}^{\text {ух м }}$ различных пунктов вдоль профиля А-В северовосточного простирания через центральные части структур ВКМ, РСП и ДДВ (рис. 5).

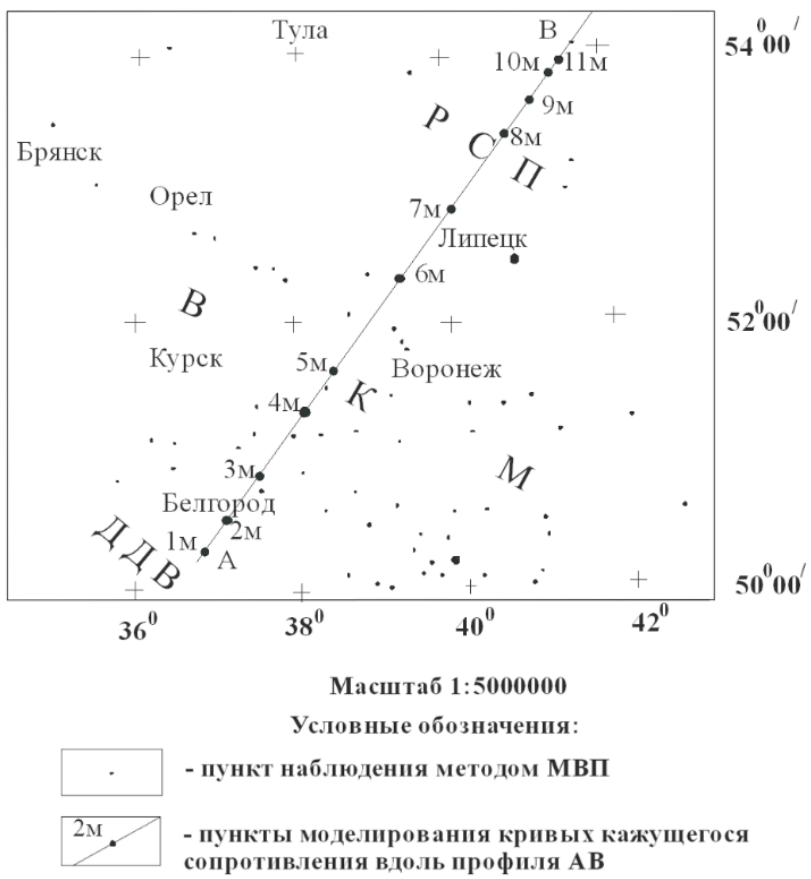

Рис. 5. Схема расположения пунктов наблюдения методом МВП и численного пленочного моделирования кривых кажущегося сопротивления.

[Fig. 5. Scheme of the location of observation points by the MEP method and numerical film modelling of apparent resistivity curves. Translation symbols from the map: Тула-Tula, Брянск
- Bryansk, Орел - Oryol, Липецк - Lipetsk, Воронеж Voronezh, Курск - Kursk, Белгород - Belgorod, Масштаб Scale, Условные обозначения - Legend, Пункт наблюдения методом МВП - Observation point by the MEP method, Пункты моделирования кривых - Points of modelling of curves.]

Полученные модельные кривые кажущегося сопротивления $\rho_{\mathrm{K}}^{\text {xу м }}$ и $\rho_{\mathrm{K}}^{\mathrm{yx}}{ }^{\mathrm{m}}$ в указанном диапазоне периодов несут информацию о влиянии региональных изменений проводимости осадочного чехла всех рассматриваемых структур. Кривые $\rho_{\mathrm{K}}{ }^{\mathrm{xy}}{ }^{\mathrm{M}}$ и $\rho_{\mathrm{K}}{ }^{\mathrm{yx}}{ }^{\mathrm{M}}$ вдоль профиля А-В смещаются по оси ординат под влиянием проводящего чехла на 4 порядка (рис. 6) $[11,12]$

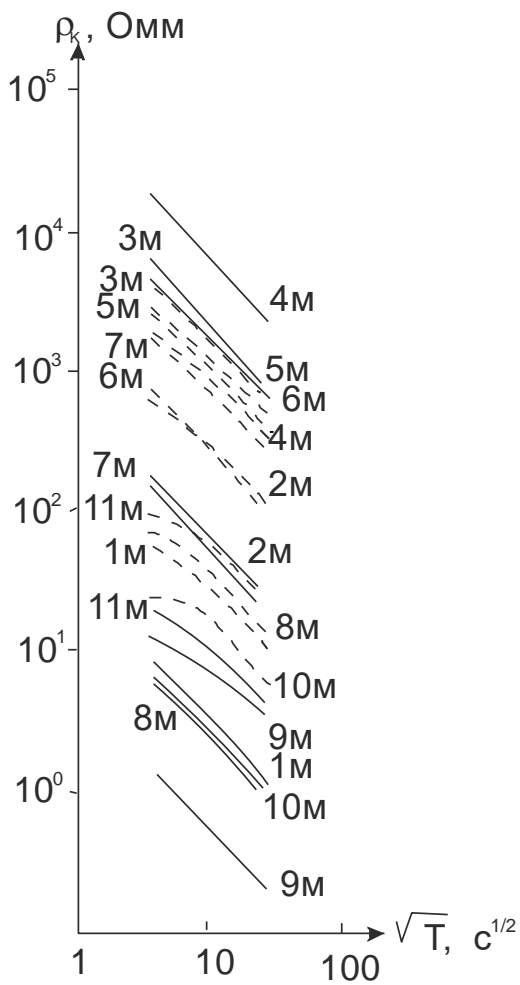

Рис. 6. Кривые кажущегося сопротивления по данным численного пленочного моделирования. Условные обозначения: кривые $\rho_{\mathrm{k}}^{\text {ху м }}-$ сплошная линия; кривые $\rho_{\mathrm{k}}^{\text {ух м }}$ пунктирная линия; $1 \mathrm{M}-11 \mathrm{M}$ - точки на профиле $\mathrm{A}$ - B, проходящем через структуры ВКМ, РСП и ДДВ в их центральной части.

[Fig. 6. Curves of apparent resistivity according to the data of numerical film modelling. Legend: curves $\rho_{\mathrm{k}^{\mathrm{xy}} \mathrm{m}}$ - solid line; curves $\rho_{\mathrm{k}}{ }^{\mathrm{yx} \mathrm{M}}-$ dotted line; $1 \mathrm{M}-11 \mathrm{M}$ - points on the A - B profile passing through the structures of the VKM, RSP, and DDV in their central part.]

На своде ВКМ модельные кривые кажущегося сопротивления $\rho_{\mathrm{K}}^{\text {ху м }}$ и $\rho_{\mathrm{K}}^{\text {ух м }}$ максимально приподняты по оси ординат до уровня 10000 Ом·м, а значения $\rho_{\mathrm{K}}^{\text {ху м }}$ на порядок превышают значения $\rho_{\kappa}^{\text {ухм }}$. На склонах ВКМ кривые кажущегося сопротивления $\rho_{\kappa}^{\text {хум }}$ и $\rho_{\kappa}^{\text {ух м }}$ смещаются по оси ординат на 2 порядка. Минимальные значения $\rho_{\mathrm{K}}^{\text {хум }}$ и $\rho_{\mathrm{K}}^{\text {ухм }}$ величиной 0.2 Ом·м наблюдаются в наиболее погруженной, центральной

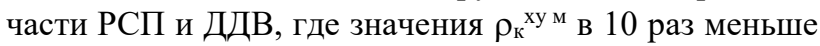


значений $\rho_{\kappa}^{\text {ух м }}[11,12]$. Для интервала периодов $900-$ 43200 с в пределах свода и склонов ВКМ отмечается слабая зависимость искажения кривых $\rho_{\mathrm{K}}^{\text {ху м }}$ и $\rho_{\mathrm{K}}^{\text {ух м }}$ от периода, что указывает на незначительные региональные индукционные эффекты, тогда как в центральной части впадин эти искажения становятся существенными.

Большинство наблюдённых кривых $\rho_{к}$ в пределах ВКМ и его склонов в разной степени занижены по уровню по сравнению с модельными. Это можно объяснить действием локальных гальванических эффектов, обусловленных влиянием проводящих отложений верхней части кристаллического фундамента.

\section{Заключение}

Выполненное численное плёночное моделирование позволило определить степень регионального влияния проводящего осадочного чехла на структуру наблюдаемого естественного электромагнитного поля. Это дает возможность провести сравнительный анализ экспериментального и модельного полей для случаев Х и Ү поляризаций первичного поля, а также получить оценку влияния осадочного чехла на низкочастотную ветвь экспериментальных кривых $\rho_{\kappa}$.

Просачиванием электрических токов в кристаллический фундамент при выполнении численного пленочного моделирования пренебрегалось, следовательно, полученный уровень кривых $\rho_{\kappa}^{\text {хум }}$ и $\rho_{\kappa}^{\text {ухм }}$ отражает не их реальное положение, а смещение вдоль оси ординат в результате действия только регионального гальванического эффекта, обусловленного осадочным чехлом.

Учитывая искажения переменного электромагнитного поля проводящим осадочным чехлом, были выявлены аномалии электропроводности по магнитовариационным и магнитотеллурическим данным в пределах ВКМ и сопряженных структур на различных уровнях земной коры, которые обусловлены объектами разной природы. Приповерхностные аномалии, вносящие искажения в наблюденное переменное электромагнитное поле, обычно связаны со спецификой петрологических ассоциаций, выполняющих наложенные геологические структуры, выявленные по данным плотностного моделирования [13 - 16].

Конфликт интересов: Автор декларирует отсутствие явных и потенциальных конфликтов интересов, связанных с публикацией настоящей статьи.

\section{ЛИТЕРАТУРА}

1. Рокитянский И. И., Логвинов И. М., Максимов В. М. Площадные магнитовариационные исследования в центральной части Русской платформы. // Изв. АН СССР, Физика Земли. 1977. № 9. С. 107-112.

2. Жданов М. С., Максимов В. М., Груздев В. Н. О результатах магнитовариационных исследований на территории ВКМ и сопредельных структур центра Русской платформы. Электромагнитные зондирования. Тез. докл. V11 Всес. школ -семинара. М., 1984. С. 75.

3. Груздев В. Н. Геоэлектрическая модель Воронежского кристаллического массива // Bonросы теории и практики геологической интерпретачии гравитационных, магнитных u электрических полей. Тез. докл. международной конференции. Воронеж, 1996. С.72-73.

4. Зингер Б. Ш., Фаинберг Э. Б. Электромагнитная индукция в неоднородных тонких слоях. М., ИЗМИРАН, 1985. 234 с.

5. Варенцов И. М., Голубев Н. Г. Программы конечно-разностного моделирования электромагнитных полей в двумерно-неоднородных средах. Алгоритмы и программы анализа геомагнитного поля. М., 1980. С. 147-163.

6. Сидоров В. А., Бучарский Б. В., Каменев Е. Н., Тикшаев В. В. Суммарная продольная проводимость водной оболочки и осадочного чехла. АН СССР, Ин-т земного магнетизма, ионосферы и распространения радиоволн. М: Наука, 1978. 12 с.

7. Файнберг Э. Б., Сидоров В. А. Суммарная продольная проводимость водной оболочки и осадочного чехла Земли. М: Наука, 1978. 624 с.

8. Жаворонкин В. И., Груздев В. Н., Фаустова Ю. С. Карта суммарной продольной электрической проводимости осадочного чехла воронежского кристаллического массива и его обрамления. Вопросы теории и практики геологической интерпретации геофизических полей. Материалы 45-й сессии Международного семинара им. Д. Г. Успенского. 2018. C. $127-128$

9. Груздев В. Н. Суммарная электропроводность консолидированной части земной коры // Вестник Воронежского государственного университета. Серия: Геология. 2014. № 2. C. $182-185$.

10. Груздев В. Н. Электропроводность территории ВКМ и сопредельных регионов. Литосфера Воронежского кристаллического массива по геофизическим и петрофизическим данным: монография, Воронеж «Научная книга», 2012. 330 с. 11. Груздев В. Н. Результаты численного пленочного моделирования электромагнитного поля в пределах воронежского кристаллического массива и сопряженных структур. Вопросы теории и практики геологической интерпретации геофизических полей. Материалы 45-й сессии Международного семинара им. Д. Г. Успенского. 2018. С. 58-59.

12. Груздев В. Н., Жаворонкин В. И. Коровые аномалии глубинной электропроводности Воронежского кристаллического массива // Вестник Воронежского государственного университета. Серия: Геология. 2015. № 1. С. 94-101.

13. Глазнев В. Н., Муравина О. М., Воронова Т. А., Холин В. М. Оценка мощности гравиактивного слоя земной коры Воронежского кристаллического массива // Вестник Воронежского государственного университета. Серия: Геология. 2014. № 4. С.78-84.

14. Муравина О. М. Плотностная модель земной коры Воронежского кристаллического массива // Вестник Воронежского государственного университета. Серия: Геология. 2016. № 1. С. 108-114.

15. Глазнев В. Н., Минц О. М., Муравина О. М. Плотностное моделирование земной коры центральной части ВосточноЕвропейской платформы // Вестн. Крауни. науки о Земле. Вып. № 29. 2016. № 1. С. 53-63.

16. Минц М. В., Глазнев В. Н., Муравина О. М. Глубинное строение коры юго-востока Воронежского кристаллического массива по геофизическим данным: геодинамическая эволюция в палеопротерозое и современное состояние коры // Вестник Воронежского государственного университета. Серия: Геология. 2017. № 4. С. 5-23. 
UDC 550.311.(470.32)

ISSN 1609-0691

DOI: https://doi.org/10.17308/geology.2021.3/3652

Received: 28.06.2021

Accepted: 02.09.2021

Published online: 07.10.2021

\title{
Numerical membranous simulation of the electromagnetic field on the Voronezh crystalline massif and adjacent structures
}

\author{
C 2021 V. N. Gruzdev ${ }^{凶}$ \\ Voronezh State University, 1, Universitetskaya pl., 394018 Voronezh, Russian Federation
}

\begin{abstract}
Introduction: Variations of the natural electromagnetic field allow studying the deep structure of the Voronezh crystalline massif and adjacent structures. Highly conductive rocks of the sedimentary cover have an effect on the nature of the observed geomagnetic field.

Methodology: We conducted numerical membranous simulation to take into account the distortions of the observed magnetotelluric field within the Voronezh crystalline massif, Ryazan-Saratov trough, and Dnieper-Donets Rift by the regional galvanic and inductive effects. The sedimentary cover of these structures was approximated by the Price-Sheinman membrane with the set total longitudinal conductivity $\mathrm{S}(\mathrm{x}, \mathrm{y})$.

Results and discussion: Under the influence of a heterogeneous conductive sedimentary cover, the horizontal components of the electric field and the vertical component of the magnetic field were seriously distorted, while the horizontal components of the variable magnetic field were significantly less subjected to the impact of the conductive sedimentary cover.

Conclusions: Taking into account the distortions of the variable electromagnetic field by the conductive sedimentary cover allowed assessing the anomalies of electric conductivity identified using geomagneticvariation and magnetotelluric data within the VCM and adjacent structures at various levels of the Earth's crust.
\end{abstract}

Keywords: Voronezh crystalline massif, numerical membranous modelling, sedimentary cover, galvanic and inductive distortions

Funding: Grant of the Russian Foundation for Basic Research 19-05-00336

For citation: Gruzdev V. N. Numerical membranous simulation of the electromagnetic field on the Voronezh crystalline massif and adjacent structures. Vestnik Voronezhskogo gosudarstvennogo universiteta. Seriya: Geologiya - Proceedings of Voronezh State University. Series: Geology, 2021, no. 3, pp. 79-86. DOI: https://doi.org/10.17308/geology.2021.3/3652

Conflict of interests: The authors declare the absence of obvious and potential conflicts of interest related to the publication of this article.

\section{REFERENCES}

1. Rokityanskii I. I., Logvinov I. M., Maksimov V. M. Ploshchadnye magnitovariatsionnye issledovaniya $\mathrm{v}$ tsentral'noi chasti Russkoi platformy. [Area magnetovariational studies in the central part of the Russian platform]. Izv. AN SSSR, Fizika Zemli - Izv. USSR Academy of Sciences, Physics of the Earth, 1977, no. 9, pp. 107-112. (In Russ.)

2. Zhdanov M. S., Maksimov V. M., Gruzdev V. N. O rezul'tatakh magnitovariatsionnykh issledovanii na territorii
VKM i sopredel'nykh struktur tsentra Russkoi platform [On the results of magnetovariational studies on the territory of the VKM and adjacent structures of the center of the Russian platform]. lektromagnitnye zondirovaniya. Tez. dokl. VII Vses. shchkol seminara. [Electromagnetic sounding. Thes. doc. VII All schools - seminar]. Moscow, 1984, p. 75. (In Russ.)

3. Gruzdev V. N. Geoelektricheskaya model' Voronezhskogo kristallicheskogo massiva [Geoelectric model of the Voronezh crystalline massif]. Voprosy teorii $i$ praktiki geologicheskoi

The content is available under Creative Commons Attribution 4.0 License.

\footnotetext{
$\bowtie$ Vladislav N. Gruzdev, e-mail: grumerr@rambler.ru
} 
interpretatsii gravitatsionnykh, magnitnykh i elektricheskikh polei. Tez. dokl. mezhdunarodnoi konferentsii. [Questions of theory and practice of geological interpretation of gravitational, magnetic and electric fields. Thes. doc. international conference] Voronezh, 1996, pp. 72-73. (In Russ.)

4. Singer B. Sh., Fainberg E. B. Elektromagnitnaya induktsiya $v$ neodnorodnykh tonkikh sloyakh [Electromagnetic induction in heterogeneous thin layers]. Moscow, IZMIRAN publ., 1985. 234 p. (In Russ.)

5. Varentsov I. M., Golubev N. G. Programmy konechnoraznostnogo modelirovaniya elektromagnitnykh polei $v$ dvumerno-neodnorodnykh sredakh. [Programs for finite-difference modeling of electromagnetic fields in two-dimensional inhomogeneous media]. Algoritmy i programmy analiza geomagnitnogo polya [Algorithms and programs for the analysis of the geomagnetic field]. Moscow, 1980, pp. 147-163. (In Russ.) 6. Sidorov V. A., Bucharskiy B. V., Kamenev E. N., Tikshaev V. V. Summarnaya prodol'naya provodimost' vodnoi obolochki $i$ osadochnogo chekhla [Total longitudinal conductivity of the water shell and sedimentary cover]. Academy of Sciences of the USSR, Institute of Terrestrial Magnetism, Ionosphere and Radio Wave Propagation. Moscow, Nauka publ., 1978. 12 p. (In Russ.) 7. Fainberg E. B., Sidorov V. A. Summarnaya prodol'naya provodimost' vodnoi obolochki i osadochnogo chekhla Zemli [Total longitudinal conductivity of the water shell and sedimentary cover of the Earth]. Moscow, Nauka publ., 1978. 624 p. (In Russ.) 8. Zhavoronkin V. I., Gruzdev V. N., Faustova Yu. S. Karta summarnoi prodol'noi elektricheskoi provodimosti osadochnogo chekhla voronezhskogo kristallicheskogo massiva i ego obramleniya [Map of the total longitudinal electrical conductivity of the sedimentary cover of the Voronezh crystalline massif and its frame]. Voprosy teorii i praktiki geologicheskoi interpretatsii geofizicheskikh polei. Materialy 45-i sessii Mezhdunarodnogo seminara im. D. G. Uspenskogo [Questions of theory and practice of geological interpretation of geophysical fields Materials of the 45th session of the International Seminar named after D. G. Uspensky]. 2018, pp. 127-128. (In Russ.)

9. Gruzdev V. N. Total Conductivity of the Consolidated Part of the Earth's Crust, Vestnik Voronezhskogo gosudarstvennogo universiteta. Seriya: Geologiya - Proceedings of Voronezh State University. Series: Geology, 2014, no. 2, pp. 182-185. (In Russ.) 10. Gruzdev V. N. Elektroprovodnost' territorii VKM i sopredel'nykh regionov. [Electrical conductivity of the VKM territory and adjacent regions]. Litosfera Voronezhskogo kristallicheskogo massiva po geofizicheskim $i$ petrofizicheskim dannym: monografiya [Lithosphere of the Voronezh crystalline massif according to geophysical and petrophysical data: monograph], Voronezh "Nauchnaya kniga" publ., 2012, 330 p. (In Russ.)

11. Gruzdev V. N. Rezul'taty chislennogo plenochnogo modelirovaniya elektromagnitnogo polya v predelakh voronezhskogo kristallicheskogo massiva i sopryazhennykh struktur. [Results of numerical film modeling of the electromagnetic field within the Voronezh crystalline massif and conjugate structures]. Voprosy teorii $i$ praktiki geologicheskoi interpretatsii geofizicheskikh polei. Materialy 45-i sessii Mezhdunarodnogo seminara im. D. G. Uspenskogo. [Problems of the theory and practice of geological interpretation of geophysical fields Materials of the 45th session of the International Seminar. D. G. Uspensky], 2018, pp. 58-59. (In Russ.)

12. Gruzdev V. N., Zhavoronkin V. I. Crustal anomalies of the deep electrical conductivity of the Voronezh crystalline massif. Vestnik Voronezhskogo gosudarstvennogo universiteta. Seriya: Geologiya - Proceedings of Voronezh State University. Series: Geology, 2015, no 1, pp. 94-101. (In Russ.)

13. Glaznev V. N., Muravina O. M., Voronova T. A., Kholin V. M. Estimation of the thickness of the gravitational layer of the earth's crust of the Voronezh crystalline massif. Vestnik Voronezhskogo gosudarstvennogo universiteta. Seriya: Geologiya - Proceedings of Voronezh State University. Series: Geology, 2014, no. 4, pp. 78-84. (In Russ.)

14. Muravina O. M. Density model of the Earth's crust of the Voronezh crystalline massif. Vestnik Voronezhskogo gosudarstvennogo universiteta. Seriya: Geologiya - Proceedings of Voronezh State University. Series: Geology, 2016, no. 1, pp. 108-114. (In Russ.)

15. Glaznev V. N., Mints O. M., Muravina O. M. Density modeling of the Earth's crust in the central part of the East European Platform [Plotnostnoe modelirovanie zemnoi kory tsentral'noi chasti Vostochno-Evropeiskoi platform]. Vestnik KRAUNTs. Nauki o Zemle, 2016, vol. 29, no. 1, pp. 53-63. (In Russ.)

16. Mints M. V., Glaznev V. N., Muravina O. M. The deep structure of the crust of the southeast of the Voronezh crystalline massif according to geophysical data: geodynamic evolution in the Paleoproterozoic and the modern state of the crust. Vestnik Voronezhskogo gosudarstvennogo universiteta. Seriya: Geologiya - Proceedings of Voronezh State University. Series: Geology, 2017. no. 4, pp. 5-23. (In Russ.)
Груздев Владислав Николаевич - к. ф.-м. н., доцент, Воронежский государственный университет, Воронеж, Российская федерация; e- mail: grumerr@ rambler.ru; ORCID http://orcid.org/0000-0002-0654-9577

Автор прочитал и одобрил окончательный вариант рукописи.
Vladislav N. Gruzdev - PhD in Physics and Mathematics, Associate Professor, Voronezh State University, Voronezh, Russian Federation; e-mail: grumerr@ rambler.ru; ORCID http://orcid.org/0000-0002-0654-9577

Author has read and approved the final manuscript. 